M066

\section{EPISODIO DI CONTAMINAZIONE DI CAMPIONI NEL SISTEMA DI LETTURA BACTEC 460 E VERIFICA CON METODICA PFGE.}

Buratta M .; Moro A.; lurlo A.; Cassetti T.; Pasquale L.; Sposini T.; Mazzolla R.; Bistoni F.

Dipartimento di Medicina Sperimentale e Sc. Biochimiche, Sez. Microbiologia, Università degli Studi di Perugia, Via del Giochetto, 06122 Perugia

Obbiettivo: abbiamo verificato, tramite elettroforesi pulsata, che l'isolamento di M. tuberculosis complex, da 5 campioni di pazienti diversi, era dovuto a contaminazione verificatasi nel Bactec 460 (BD).

Materiali e Metodi: i campioni, provenienti da pazienti ricoverati in varie Cliniche dell'Azienda Ospedaliera di Perugia, sono pervenuti nel nostro Laboratorio di Micobatteriologia ed hanno subito il normale iter diagnostico di sbatterizzazione e semina. Sono stati seminati in terreno solido Lowenstein Jensen e in terreno liquido $7 \mathrm{H} 12$ Middlebrook (12 B) del sistema radiometrico Bactec 460 (BD). Dalle colture sviluppatesi è stata eseguita la tipizzazione con il test AccuProbe (GenProbe). Tali colonie sono state inserite in piccoli gel di agarosio, sottoposti a trattamenti litici con distruzione della parete cellulare e liberazione di DNA cromosomico. Il DNA è stato digerito con l'enzima di restrizione XbAI e sottoposto ad elettroforesi in campo pulsato (PFGE).

Risultati: la colorazione Ziehl Neelsen allestita sui campioni era positiva solo per il paziente $\mathrm{n}^{\circ} 1$ e negativa per gli altri 4. Dai 5 campioni seminati in $12 \mathrm{~B}$ sono stati isolati $5 \mathrm{M}$. tuberculosis complex. L'osservazione delle semine dirette dei campioni in terreno solido Lowenstein Jensen ha mostrato crescita solo nel campione $n^{\circ} 1$. Notizie cliniche riferivano malattia tubercolare solo nel paziente $\mathrm{n}^{\circ} 1$.

Il risultato dell'elettroforesi in campo pulsato ha mostrato profili genomici identici in tutti e $5 \mathrm{i}$ campioni.

Conclusioni: la contaminazione del terreno liquido dei 4 campioni negativi si è verificata a causa di un cattivo funzionamento del sistema di sterilizzazione degli aghi per la lettura dei flaconi 12B del Bactec 460. L'elettroforesi pulsata ha messo in evidenza che i profili genomici dei 5 micobatteri erano identici e quindi derivanti da un unico microorganismo. Le notizie cliniche hanno confermato l'avvenuta contaminazione dimostrando l'importanza della valutazione critica da parte dell'operatore dei dati di laboratorio correlati ai dati clinici.

\section{M067}

\section{VERIFICA DI VITALITA' DEI BACILLI TUBERCOLARI IN PREPARATI MICROSCOPICI PER LA COLORAZIONE DI ZIEHL NEELSEN.}

Moro A.; Buratta M.; lurlo A.; Cassetti T.; Pasquale L.; Sposini T.; Mazzolla R.; Bistoni F

Dipartimento di Medicina Sperimentale e Sc. Biochimiche, Sez. Microbiologia, Università degli Studi di Perugia, Via del Giochetto, $06 / 22$ Perugia

Obbiettivo: la preparazione di vetrini da campioni per la colorazione Ziehl Neelsen, rappresenta una potenziale fonte di infezione poiché i bacilli sopravvivono se non opportuna- mente trattati prima della colorazione. Abbiamo valutato la vitalità dei bacilli dopo vari trattamenti di inattivazione. Materiali e Metodi: a $10 \mathrm{ml}$ di espettorato autoclavato è stato aggiunta una sospensione di M. tuberculosis H37Ra, ATCC 25177, per raggiungere le concentrazioni di 0,5 McF, $1 \mathrm{McF}$ e $2 \mathrm{McF}$. I vetrini, sterilizzati, sono stati preparati con le sospensioni batteriche e lasciati asciugare sotto cappa. I trattamenti di inattivazione utilizzati sono stati : calore $\left(80^{\circ} \mathrm{C}\right)$; esposizione ai raggi ultravioletti e fenolo al $5 \%$. I tempi di esposizione per il calore, gli UV ed il fenolo al 5\% sono stati : $30 \mathrm{~min} ., 1 \mathrm{~h}, 2 \mathrm{~h}$ e $24 \mathrm{~h}$. I vetrini sono stati incubati a $37^{\circ} \mathrm{C}$ per 10 giorni in tubi Falcon contenenti $20 \mathrm{ml}$ di 7 H9 Middlebrook. Terminata l'incubazione, dai brodi centrifugati, i sedimenti sono stati seminati in MGIT 9600 ed incubati 8 settimane.

Risultati: Dopo $2 \mathrm{~h}$ in stufa a $80^{\circ} \mathrm{C}$ tutte e tre le sospensioni di micobatteri risultavano non vitali. Gli UV sono in grado di inattivarle solo dopo $24 \mathrm{~h}$ di esposizione. Al contrario, il fenolo al $5 \%$, dopo un contatto con la sospensione batterica per 30 minuti è in grado di uccidere tutti i bacilli presenti. Conclusioni: l'esposizione al calore ad $80^{\circ} \mathrm{C}$ per $2 \mathrm{~h}$ ed il contatto per 30 minuti con il fenolo al 5\% rappresentano il tempo minimo necessario per inattivare i bacilli tubercolari, alternativamente una buona efficacia è dimostrata dal trattamento con i raggi UV dopo $24 \mathrm{~h}$ di esposizione. La tempestività della risposta microscopica consente al clinico una più rapida diagnosi e terapia antibiotica adeguata. Questo studio ci consente di associare la rapidità dell'esame diretto con una buona sicurezza di laboratorio.

\section{M068 \\ RICERCA DIRETTA DI M. TUBERCULOSIS IN CAMPIONI RESPIRATORI CON IL SISTEMA AUTOMATICO BDPROBETEC ET.}

Di Taranto A., De Nittis R.,* Mosca A., *Barra Parisi G., Antonetti R.,* Miragliotta G.

Laboratorio di Microbiologia, Ospedali Riuniti di Foggia,

* Cattedra di Microbiologia, Dip. MIDIM, Università di Bari

BDProbeTec ET (Becton Dickinson) è un sistema automatico che permette la ricerca rapida di $M$. tuberculosis direttamente nel campione clinico. Questo sistema utilizza come amplificazione isotermica del DNA la tecnologia SDA (Strand Displacement Amplification) e, come metodo di rilevamento, il trasferimento di energia fluorescente, consentendo l'amplificazione e la rivelazione del DNA in real-time. Presso il laboratorio di Microbiologia degli Ospedali Riuniti di Foggia, per la ricerca diretta di M. tuberculosis, al tradizionale esame batterioscopico e colturale (Bactec 960 TB, Becton Dickinson) è stato affiancato il sistema SDA. Nell'anno 2002 sono stati esaminati 712 campioni respiratori tutti provenienti da pazienti ospedalizzati ed ambulatoriali sui quali di routine viene eseguita la ricerca di M. tuberculosis. In particolare, sono stati processati 76 espettorati, 596 BAL e broncoaspirati, 40 liquidi pleurici.

16/21 (76.2\%) campioni positivi all'esame colturale sono risultati positivi col test SDA. In particolare, all'esame batterioscopico 12/21 sono risultati positivi e 4/21 negativi. 5/21 $(23.8 \%)$ campioni positivi all'esame colturale sono risultati negativi al batterioscopico e al test SDA. Infine,5 campioni negativi all'esame batterioscopico e colturale sono risultati positivi al test SDA (4 da pazienti in terapia antitubercolare). I nostri risultati suggeriscono che la real-time PCR, metodica di semplice esecuzione ,è attendibile per la rilevazione diretta di M. tuberculosis da campione clinico e può essere 